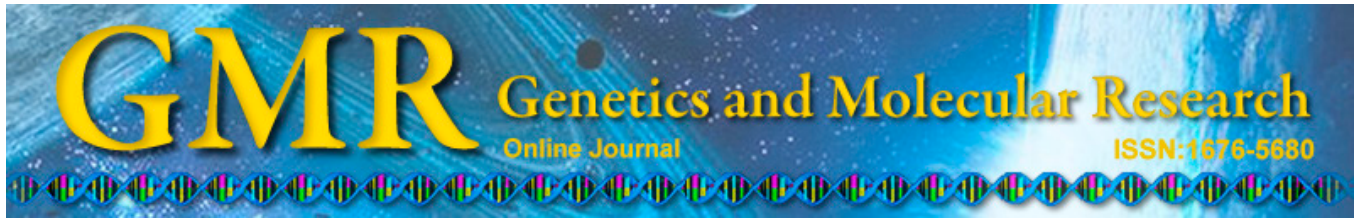

\title{
Molecular cloning, characterization, and expression of Rab5B, Rab6A, and Rab7 from Litopenaeus vannamei (Penaeidae)
}

\author{
Y.Z. Zhao*, X.L. Chen*, D.G. Zeng, C.L. Yang, M. Peng and X.H. Chen \\ Guangxi Key Laboratory of Aquatic Genetic Breeding and Healthy Aquaculture, \\ Guangxi Academy of Fisheries Sciences, Nanning, China \\ *These authors contributed equally to this study. \\ Corresponding author: X.H. Chen \\ E-mail: gxnnscs@126.com
}

Genet. Mol. Res. 14 (3): 7740-7750 (2015)

Received October 28, 2014

Accepted April 6, 2015

Published July 13, 2015

DOI http://dx.doi.org/10.4238/2015.July.13.20

\begin{abstract}
The Rab protein family belongs to a superfamily of ras-like GTP-binding proteins. Rab proteins regulate many steps of membrane trafficking. In this study, three Rab family members, Rab5B, Rab6A, and Rab7, designated LvRab5B, LvRab6A, and LvRab7, were cloned from Litopenaeus vannamei. The full-length cDNA sequences of LvRab5B, LvRab6A, and LvRab7 were 1383, 873, and 767 nucleotides in length and they encoded proteins of 211, 212, and 205 amino acids, respectively. Using qRT-PCR, the mRNA expression levels of the three proteins were determined in the hepatopancreas of $L$. vannamei at different stages after infectious hypodermal and hematopoietic necrosis virus and white spot syndrome virus challenge. The results indicated that the mRNA expression levels of LvRab5B, LvRab6A, and LvRab7 were all significantly up-regulated after virus injection, suggesting that these genes may play essential roles in the immune response to viral infection in shrimp.
\end{abstract}

Key words: Litopenaeus vannamei; Rab5B; Rab6A; Rab7 


\section{INTRODUCTION}

Cell growth, development, division, biosynthesis, secretion, and other biological processes are accompanied by protein transport between the organelles. In cells, protein transport is accomplished by vesicle transport. Rab proteins can act as molecular switches of vesicle transport to play a key role in vesicle formation, transport, adhesion, and anchoring (Hutagalung and Novick, 2011). The Rab is the largest subfamily of the small GTP-binding proteins (Pereira-Leal and Seabra, 2001). Recent studies have found that some Rab family members, including Rab7, can specifically recognize endosomal vesicles, and conduct lysosomal transport by mediating the fusion of endosomal vesicles and lysosomes (Jordens et al., 2006). When viruses enter the host cells, they combine with Rab proteins to avoid lysosomal digestion (Zhang et al., 2009).

The Pacific white shrimp, Litopenaeus vannamei, has become the leading farmed species in the western hemisphere due to its high yield potential, low salinity requirements, and strong resistance to disease (Valles-Jimenez et al., 2004). Recently, viral diseases have severely threatened the shrimp aquaculture industry. Among the viral diseases, white spot syndrome virus (WSSV), infectious hypodermal and hematopoietic necrosis virus (IHHNV), and Taura syndrome virus (TSV) have caused serious economic losses to the shrimp industry worldwide (Lightner et al., 1997). To date, no effective cure for these diseases has been found. Shrimp lack an acquired immune system and instead they rely on very efficient innate immune systems. An understanding of the Rab proteins in shrimp and their responses against pathogens will promote the development of effective techniques to prevent viral diseases of shrimp (Robalino et al., 2007).

Given the importance of Rab proteins in the shrimp immune response, we cloned the full-length cDNA sequences of Rab5B, Rab6A, and Rab7 from L. vannamei, and analyzed their characteristics, evolution, tissue distribution, and differential expression in response to viral infection. This paper is the first report on cloning full-length cDNA sequences of Rab5B, Rab6A, and Rab7 in L. vannamei, and will promote the development of effective techniques to prevent viral diseases of shrimp.

\section{MATERIAL AND METHODS}

\section{Cloning of the Rab5B, Rab6A, and Rab7 proteins from L. vannamei}

Partial cDNA sequences that are homologous to Drosophila Rab5B, Rab6A, and Rab7 were retrieved from the sequenced L. vannamei transcriptome data, which was previously completed in our research group (Zeng et al., 2013). The full length cDNA sequences of shrimp Rab5B, Rab6A, and Rab7 were then obtained using the rapid amplification of cDNA ends (RACE) method. Briefly, total RNA was extracted from L. vannamei hepatopancreas using Trizol reagent (GibcoBRL, USA). The cDNA templates for RACE-PCR were prepared using the PrimeScript RT kit (TaKaRa, Japan). The 5'-RACE and 3'-RACE-nested-PCR amplifications were performed with 5'-RACE primers, 3'-RACE primers, and Rab5B, Rab6A, and Rab7 specific reverse primers (Table 1). The second PCR products were individually cloned into pMD-20T vectors (TaKaRa), and 30 positive clones were randomly selected and sequenced (performed by Tiangen Company, China). 
Table 1. Primers for 5'-RACE and 3'-RACE-nested-PCR amplifications of Rab5B, Rab6A, and Rab7 in Litopenaeus vannamei.

\begin{tabular}{llll}
\hline Gene & Specific primers & Application & Sequence (5'-3') \\
\hline Rab5B & R5B3R1 & 3'-RACE outer & TGGTGAATCAGCAGTCGGAAAGTCTAC \\
& R5B5R1 & CACTCTTTGGCAGTTTCTTTGCTATTG \\
& R5B3R2 & 5'-RACE outer & TTGGGACACGGCTGGTCAAGAACG \\
& R5B5R2 & 3'-RACE inner & AACCATCCTCTTGTTGGCTAAATCTGC \\
Rab6A & R6A3R1 & 5'-RACE inner & GCGAACAGAGCGAGGCAGTGATG \\
& R6A5R1 & 3'-RACE outer & CGGATATATGAGGGAATAAGGCTGCGG \\
& R6A3R2 & 5'-RACE outer & GGTCTCCACGGAGGAAGGTGAACG \\
& R6A5R2 & 3'-RACE inner & CGATGCCGATGGTCGCCTGGTAG \\
Rab7 & R73R1 & 5'-RACE inner & GGAGAATAGGGCGGTATCGACGAAGC \\
& R75R1 & 3'-RACE outer & CGCTTCGTCGATACCGCCCTATTCTC \\
& R73R2 & 5'-RACE outer & CAACAAGGCTAACAGGATGCGTGC \\
& R75R2 & 3'-RACE inner & CGGTCCCTTGGTGAGGCTTGAATTAG \\
\hline
\end{tabular}

\section{Bioinformatic analysis}

The open reading frame (ORF) and DNA translation were determined using the NCBI ORF Finder program (http://www.ncbi.nlm.nih.gov/projects/gorf/). The molecular weights $(\mathrm{Mw})$ and the theoretical isoelectric points (pI) of Rab5B, Rab6A, and Rab7 were calculated using the $\mathrm{pI} / \mathrm{Mw}$ tool (http://web.expasy.org/compute_pi/). To determine the protein family, the structural domains and functional sites of the polypeptide sequences were analyzed by searching the Pfam database (http://pfam.xfam.org/). Multiple sequence alignment was performed using the ClustalW2 software (http://www.ebi.ac.uk/Tools/msa/clustalw2/).

\section{Western blot analysis of recombinant Rab5B, Rab6A, and Rab7 proteins}

The coding sequences of Rab5B, Rab6A, and Rab7 were individually cloned into the pEASY ${ }^{\mathrm{TM}}$-E2 vector (TransGen Biotech, China) with a His-tag at the end of the cloned sequences and transformed into Escherichia coli BL21 (DE3) strain (Novagen, USA), which were subsequently induced by $0.1 \mathrm{M}$ isopropyl- $\beta$-D-thiogalactosidase for expression of recombinant proteins. Bacterial cells were harvested and sonicated, and the inclusion bodies were obtained, washed with $2 \mathrm{M}$ urea in PBS buffer, and dissolved in $8 \mathrm{M}$ urea. The Histagged recombinant proteins were purified by MagExtractor His-Tag NPK-700 (Japan) according to the manufacturer protocol. The purified proteins were analyzed by western blot using mouse anti-6His antibody (TransGen Biotech) as the primary antibody.

\section{Quantitative real-time PCR (qRT-PCR) analysis}

Specific pathogen-free L. vannamei shrimp were obtained from the National and Guangxi Shrimp Genetic Breeding Center (Guangxi Province, China), with an average body weight of approximately $20 \mathrm{~g}$. The shrimp were maintained in 1000-L glass seawater tanks $\left(25^{\circ}-26^{\circ} \mathrm{C}\right)$ to acclimatize for 5 days before the experiment. In the challenge experiment, shrimp in the treatment groups were injected with $10 \mu \mathrm{L}$ IHHNV suspension $(\sim 100 \mathrm{IHHNV}$ copies $/ \mu \mathrm{L})$ and $10 \mu \mathrm{L}$ WSSV suspension $(\sim 100 \mathrm{WSSV}$ copies $/ \mu \mathrm{L})$, and prepared as described by Prior et al. (2003). In parallel, the shrimp in the control group were injected with $10 \mu \mathrm{L}$ PBS. At $0,2,4,6,8,12,24,36,48,72,84$, and $108 \mathrm{~h}$ post-injection, the tissues of heart, gills, 
intestine, stomach, hepatopancreas, and muscle of shrimp were isolated from three individuals in each group and frozen in liquid nitrogen.

Total RNA was extracted using Trizol reagent (Gibcobrl) following the manufacturer protocol, and DNA was removed using DNase (Gibcobrl). RNA purity was checked using a nucleic acid analyzer (Bio-Rad, USA) and integrity was detected using $1.5 \%$ agarose gel electrophoresis. RNA was reverse transcribed into cDNA using a PrimeScript RT kit (TaKaRa). The primers (Table 2) for qRT-PCR were designed according to the Rab5B, Rab6A, and Rab7 sequences. The primers for $18 \mathrm{~S}$ rRNA (used as an internal reference gene) were 5'-CTCTGCTGAACCGCATTACTTG-3' (forward) and 5'-TGCCGAGGGTTTTGGTCT-3' (reverse). The PCR system consisted of $2 \mu \mathrm{L}$ cDNA template, $12.5 \mu \mathrm{L} 2 \mathrm{X}$ SYBR Premix Ex Taq II Buffer, $1 \mu \mathrm{L}$ Primer Mix $(10 \mu \mathrm{M})$, and water to a total volume of $20 \mu \mathrm{L}$. PCRs were performed in triplicate in a 9700 thermal cycler (ABI, USA) under the following conditions: $95^{\circ} \mathrm{C}$ for $30 \mathrm{~s}$, followed by 40 cycles of $95^{\circ} \mathrm{C}$ for $5 \mathrm{~s}$ and $60^{\circ} \mathrm{C}$ for $30 \mathrm{~s}$.

Table 2. Primers for qRT-PCR analysis of Rab5B, Rab6A, and Rab7 in Litopenaeus vannamei.

\begin{tabular}{ll}
\hline Primer name & Sequence $\left(5^{\prime}-3^{\prime}\right)$ \\
\hline RAB5B-F & GGAGACCTCTGCTAAGACTGCTATG \\
RAB5B-R & CTGTGCTGGCTGGTTATTGG \\
RAB6A-F & ATGCAAATCCTTCCACCAAAC \\
RAB6A-R & CCTTTGCTTTTCGTCACCTTC \\
RAB7-F & GACCCTGACCACTTCCCATTT \\
RAB7-R & CATTGTTGTGCTCGCTTCGT \\
\hline
\end{tabular}

\section{RESULTS}

\section{Cloning and sequence analysis of shrimp Rab5B, Rab6A, and Rab7 (LvRab5B, LvRab6A, and LvRab7)}

Based on the partial cDNA sequences that were retrieved from the L. vannamei transcriptome data, three sets of gene specific-primers were designed to amplify the full-length cDNA sequences of LvRab5B, LvRab6A, and LvRab7 using the 3'- and 5'-RACE techniques (Figure 1). Three sequences, 1383, 873, and $767 \mathrm{bp}$ in length, representing the complete cDNA sequences of LvRab5B, LvRab6A, and LvRab7, respectively, were obtained. The cDNA sequences have been submitted to GenBank under the accession Nos. JQ901103, JX073679, and JQ581679. The fulllength cDNA sequences of LvRab5B, LvRab6A, and LvRab7 contain ORFs of 633, 636, and 615 bp, encoding 211, 212, and 205 amino acid proteins with calculated Mw of 22.969, 23.751, and $23.330 \mathrm{kDa}$ and $\mathrm{pI}$ of 8.131, 5.568, and 5.850, respectively. Figure 1 shows the deduced amino acid sequences of LvRab5B, LvRab6A, and LvRab7. Importantly, all these newly cloned proteins have four conserved GTP-binding or GTPase regions of the small G protein superfamily (G1, G3, G4, and G5), as well as an effector site (G2) (Figure 2). These five regions are characteristic of Rab proteins (Bourne et al., 1990), suggesting that LvRab5B, LvRab6A, and LvRab7 may be the active GTPase that can cycle between the GDP- and GTP-bound states.

To determine the protein family, the LvRab5B, LvRab6A, and LvRab7 polypeptide sequences were analyzed by searching the Pfam database. The results showed that their amino acid sequences contain a Pfam profile CL0023 (Rab), indicating that LvRab5B, LvRab6A, and LvRab7 belong to the Rab protein family. Furthermore, the full length amino acid sequences 
of LvRab5B, LvRab6A, and LvRab7 and some Rab members from Drosophila were subjected to phylogenetic analysis using ClustalW2 (Figure 3). According to the phylogenetic tree, the LvRab5B, LvRab6A, and LvRab7 are mostly clustered to the Drosophila Rab5, Rab6, and Rab7, respectively. This also demonstrates that LvRab5B, LvRab6A, and LvRab7 clearly belong to the Rab protein family.

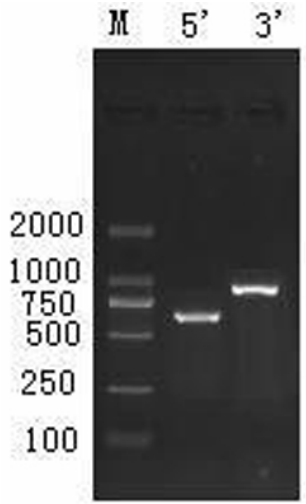

LvRab5B

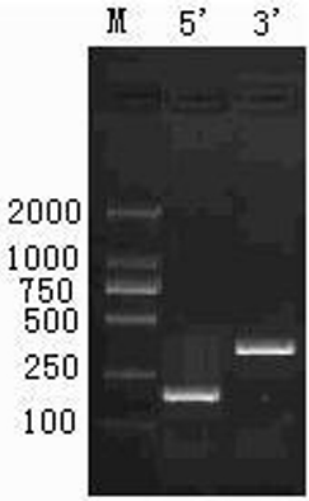

LvRab6A

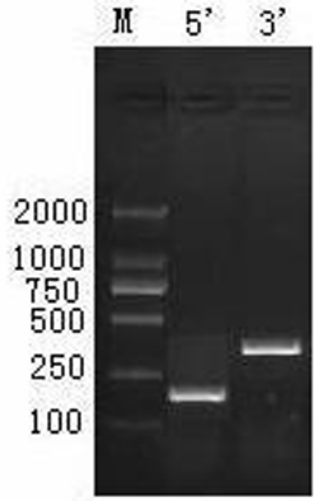

LvRab?

Figure 1. PCR amplification products of LvRab5B, LvRab6A, and LvRab7 from the 3'- and 5'-RACE techniques. Lane $M=2000$-bp DNA ladder marker; lane $5^{\prime}=$ PCR amplification products of the $5^{\prime}$-RACE; lane $3^{\prime}=$ PCR amplification products of the 3' RACE.

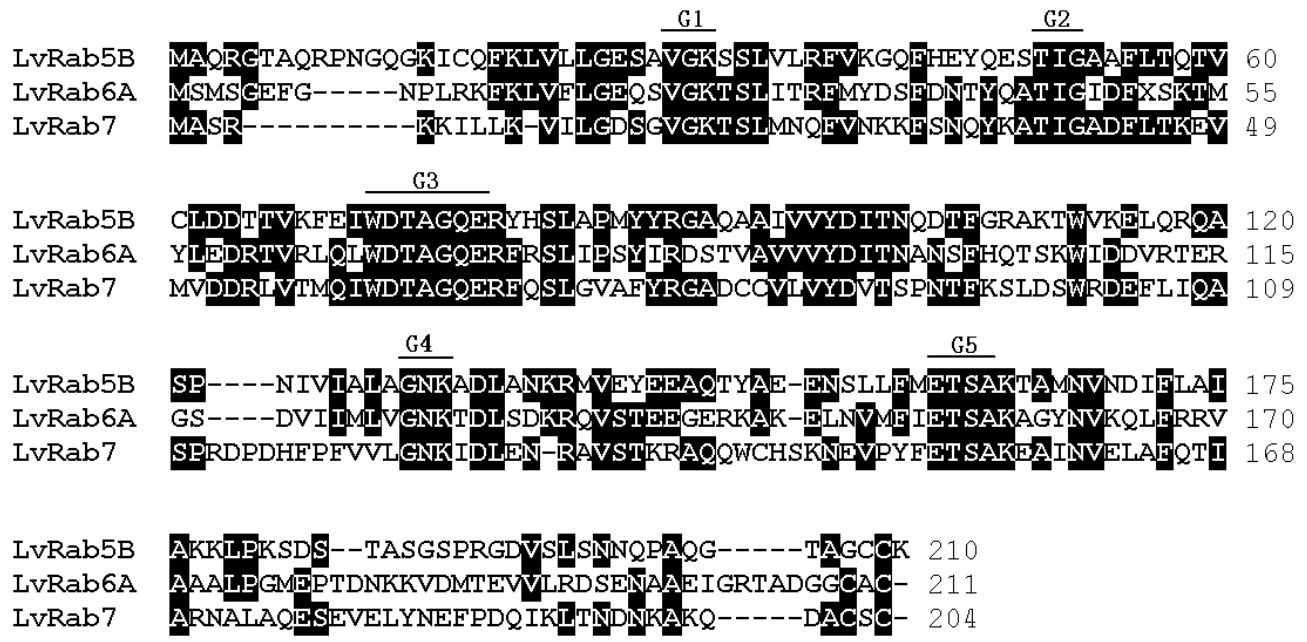

Figure 2. Alignment of LvRab5B, LvRab6A, and LvRab7 from Litopenaeus vannamei on the basis of their amino acid sequences. Identical amino acids found in all sequences are presented with a black background. G1, G3, G4, and G5 represent four conserved GTP-binding or GTPase regions of the small G protein superfamily; G2 represents an effector site. 


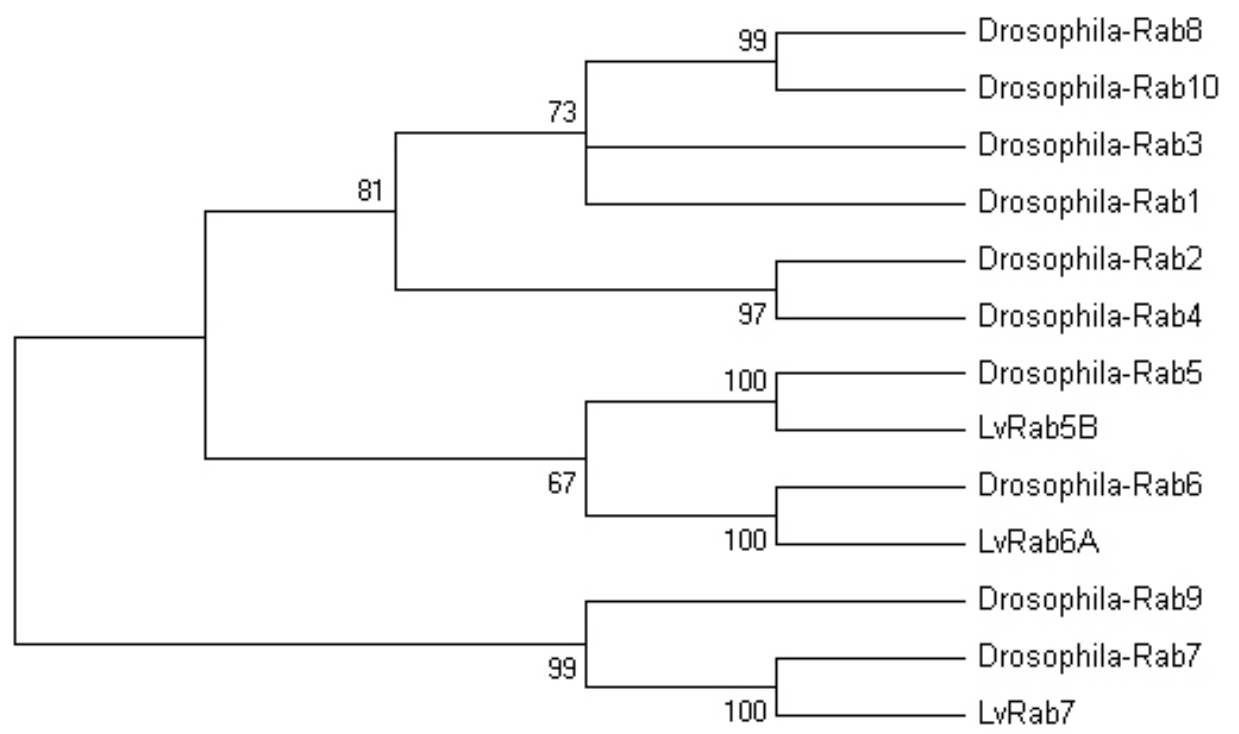

Figure 3. Phylogenetic tree showing the relationship between LvRab5B, LvRab6A, and LvRab7 amino acid sequences with those of several other known Rab members from Drosophila. The reported Drosophila Rab amino acid sequences were from GenBank and the accession Nos. are as follows: Drosophila-Rab1 (NP_732610), Drosophila-Rab2 (BAA21706), Drosophila-Rab3 (AAF58762), Drosophila-Rab4 (BAA88243), Drosophila-Rab5 (AAN85553), Drosophila-Rab6 (BAA21707), Drosophila-Rab7 (BAA88245), Drosophila-Rab8 (BAD07038), Drosophila-Rab9 (NP_609966), Drosophila-Rab10 (AAF50924). Numbers on the branches represent bootstrap support for 2000 replicates.

\section{Western blot analysis of recombinant LvRab5B, LvRab6A, and LvRab7 proteins}

The coding sequences of LvRab5B, LvRab6A, and LvRab7 were individually subcloned into a pEASYTM-E2 vector with His-tag at the end of the cloned sequences and transformed into E. coli BL21 (DE3) strain. Following induction, purification, and western blot analysis of the purified proteins using monoclonal antibody against the His-tag, each of the expressed proteins was recovered as a single protein band, which was consistent with the Mw calculated from the corresponding deduced amino acid sequence (Figure 4). The result confirmed the identification of LvRab5B, LvRab6A, and LvRab7.

\section{Expressions of LvRab5B, LvRab6A, and LvRab7 during normal conditions and after IHHNV and WSSV challenge conditions}

To study the tissue expression of LvRab5B, LvRab6A, and LvRab7, their mRNA expression levels were determined in various tissues of $L$. vannamei before virus challenge using qRT-PCR. The $18 \mathrm{~S}$ rRNA of $L$. vannamei was used as a housekeeping control gene. The results of qRT-PCR showed that the LvRab5B, LvRab6A, and LvRab7 were expressed in all tissues tested, including heart, gill, intestine, stomach, hepatopancreas, and muscle, with the highest expression level in the intestine. In addition, a particularly high expression level of LvRab6A was found in intestine (Figure 5). 


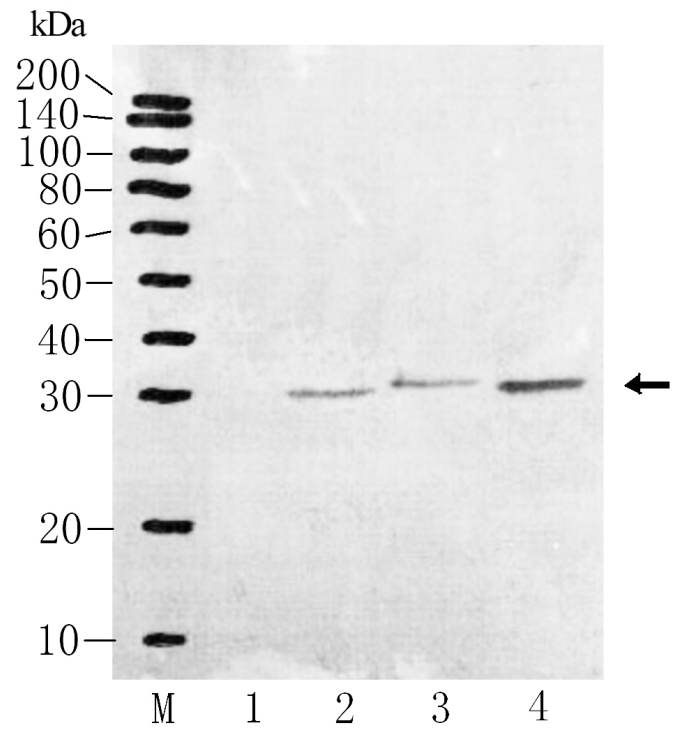

Figure 4. Western blot analysis with monoclonal anti-His antibodies. Lane $M=$ molecular size standards; lane 1 = uninduced culture; lane $2=\mathrm{LvRab} 5 \mathrm{~B}$; lane $3=\mathrm{LvRab} 6 \mathrm{~A}$; lane $4=\mathrm{LvRab} 7$. The fusion proteins are indicated by an arrow.

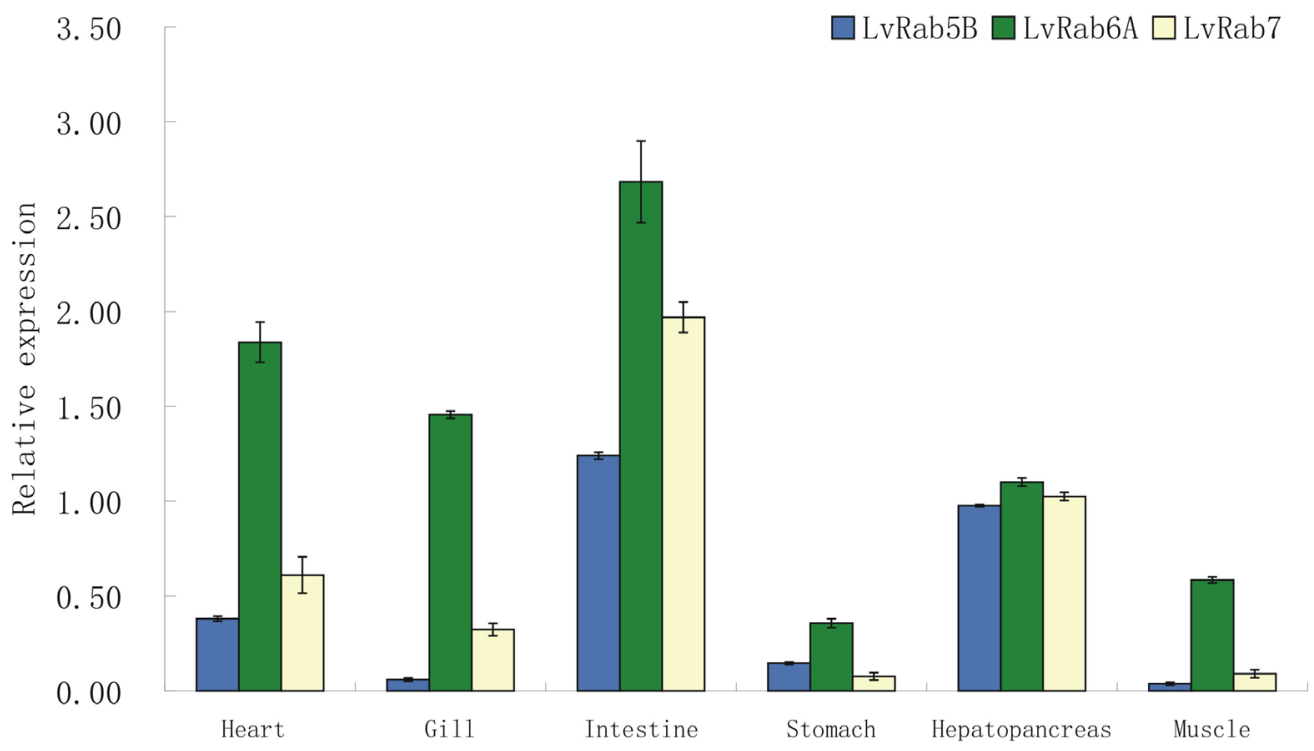

Figure 5. LvRab5B, LvRab6A, and LvRab7 mRNA expression in various tissues from Litopenaeus vannamei before viral infection. The 18S rRNA gene was used as an internal control for all tissues. The y-axis shows the relative expression level (means $\pm 2 \mathrm{SD}, \mathrm{N}=3$ ). 
To determine the participation of LvRab5B, LvRab6A, and LvRab7 in the innate immune response in shrimp, their mRNA expression levels were determined in the hepatopancreas of $L$. vannamei at different stages after IHHNV and WSSV challenge using qRT-PCR. The hepatopancreases of shrimp were collected at $0,2,4,6,8,12,24,36,48,72,84$, and 108 $h$ post-virus injection. The qRT-PCR showed that the mRNA expression levels of LvRab5B, LvRab6A, and LvRab7 following IHHNV and WSSV challenge were similar, with expression levels mostly down-regulated during $0-36 \mathrm{~h}$ post injection, and then significantly up-regulated during 48-84 h post-injection, and finally down-regulated during the $108 \mathrm{~h}$ post injection phase (Figures 6-8). These results suggest that LvRab5B, LvRab6A, and LvRab7 may play essential roles in the immune response to viral infection in shrimp.

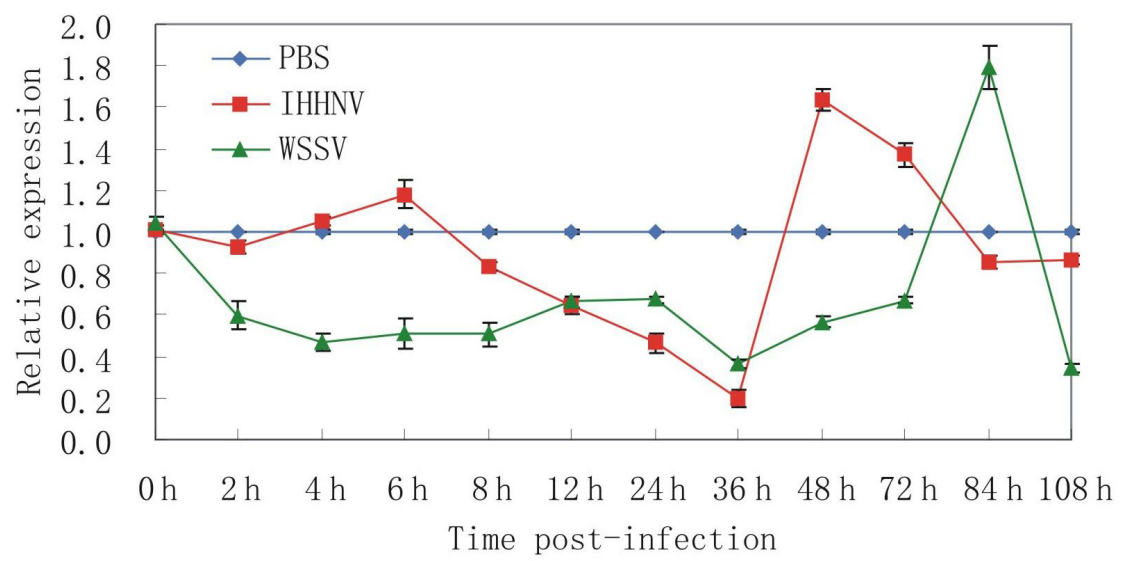

Figure 6. LvRab5B expression in the hepatopancreas of Litopenaeus vannamei after IHHNV and WSSV challenge. The $\mathrm{x}$-axis shows hours post virus challenge and the $\mathrm{y}$-axis shows the relative expression levels of LvRab5B (means $\pm 2 \mathrm{SD}, \mathrm{N}=3$ ).

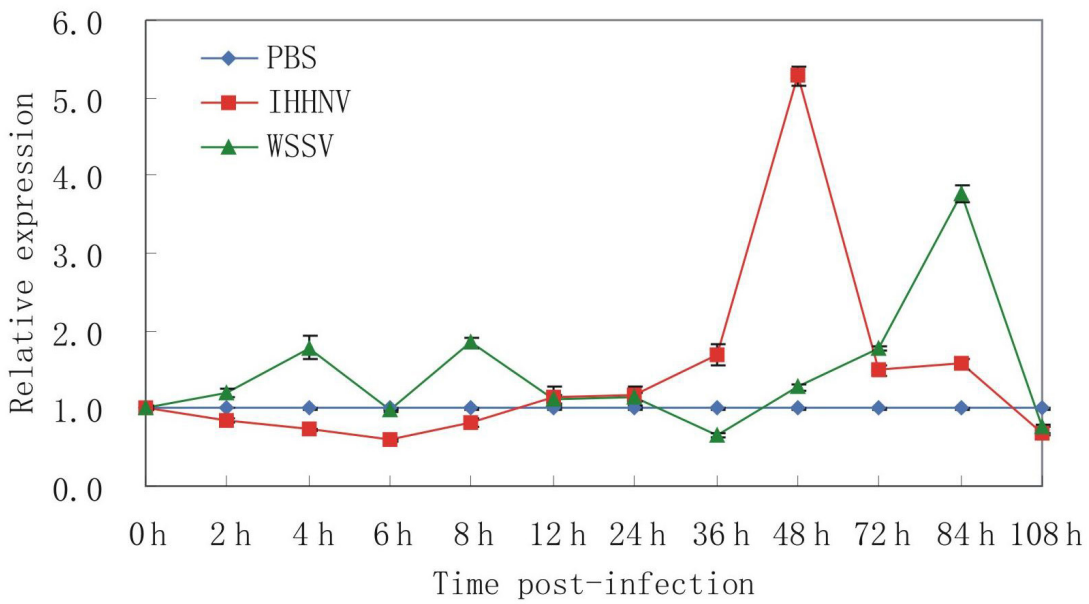

Figure 7. LvRab6A expression in the hepatopancreas of Litopenaeus vannamei after IHHNV and WSSV challenge. The $\mathrm{x}$-axis shows hours post virus challenge and the $\mathrm{y}$-axis shows the relative expression levels of LvRab6A (means $\pm 2 \mathrm{SD}, \mathrm{N}=3$ ). 


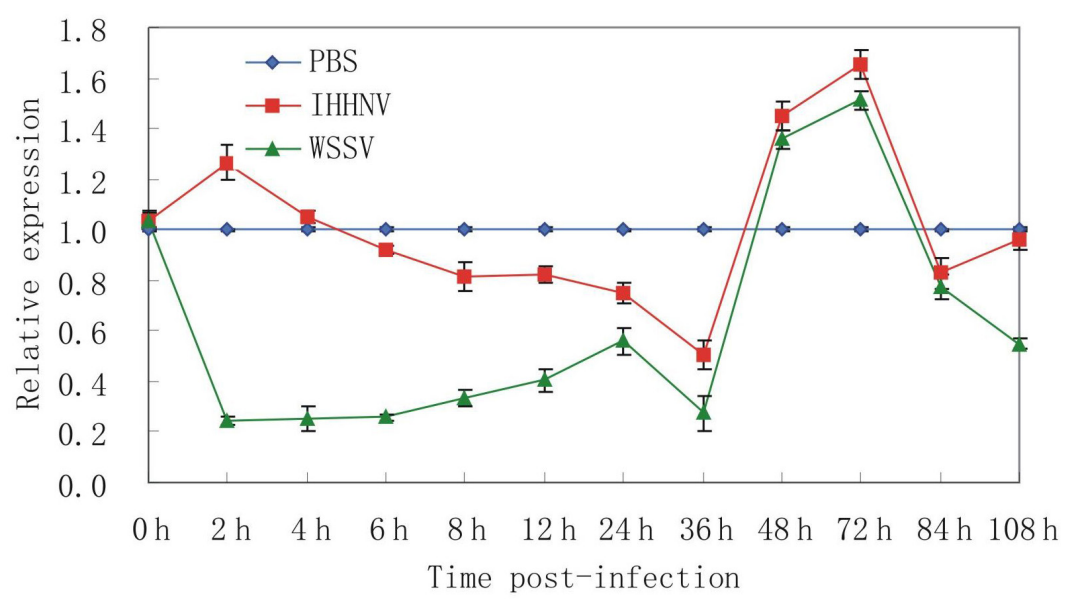

Figure 8. LvRab7 expression in the hepatopancreas of Litopenaeus vannamei after IHHNV and WSSV challenge. The $\mathrm{x}$-axis shows hours post virus challenge and the $\mathrm{y}$-axis shows the relative expression levels of LvRab7 (means $\pm 2 \mathrm{SD}, \mathrm{N}=3$ ).

\section{DISCUSSION}

Rab proteins play a crucial role in vesicle formation, transport, adhesion, and anchoring by acting as molecular switches of vesicle transport in animal cells (Segev, 2001; Hutagalung and Novick, 2011). Rab proteins are present in all eukaryotes and they have been highly conserved during eukaryote evolution, while exhibiting functional diversity and a number of differences in different species (Saito-Nakano et al., 2005). Currently, more than sixty types of Rab proteins, mainly in model organisms, have been identified. In Drosophila, 26 types of Rab proteins have been cloned and in humans, more than 60 types of Rab proteins have been cloned (Diekmann et al., 2011). However, only two types of Rab proteins, Rab6 and Rab7, have been identified in shrimp, to date. In this study, we identified, for the first time, the full-length cDNA sequences of LvRab5B, LvRab6A, and LvRab7 from L. vannamei. To our knowledge, LvRab5B is the first Rab homologue from crustaceans to be identified and characterized.

Sequence analysis showed that the newly cloned LvRab5B, LvRab6A, and LvRab7 belong to the Rab protein family. Western blot analysis also demonstrated that the Mw of recombinant LvRab5B, LvRab6A, and LvRab7 proteins were consistent with the Mw calculated from the amino acid sequences deduced. We compared the amino acid sequences of LvRab5B, LvRab6A, and LvRab7 with some other Rab members of Drosophila, and with the results from searching the Pfam database, these demonstrate that LvRab5B, LvRab6A, and LvRab7 clearly belong to the Rab protein family. Sequence alignments showed that the major domains of LvRab5B, LvRab6A, and LvRab7 are similar to those in other species, revealing high conservation of the main functional domains between different species. This suggests that the biological functions of LvRab5B, LvRab6A, and LvRab7 may be similar to the corresponding Rab members in other model organisms.

To study the tissue expression of the newly identified Rab proteins, their mRNA expression levels were determined using qRT-PCR in heart, gill, intestine, stomach, hepa- 
topancreas, and muscle tissues of $L$. vannamei before virus challenge. The results showed that LvRab5B, LvRab6A, and LvRab7 were expressed in all tissues tested, with the highest expression level in the intestine. Since intestine is an important tissue related to the absorption and transport of nutrients, intracellular transport is more active here than in other tissues (Martin et al., 2006). This might explain the high expression levels of LvRab5B, LvRab6A, and LvRab7 in the intestine.

IHHNV and WSSV are the main viral pathogens for shrimp that lead to great economic losses to the shrimp farming industry (Tang et al., 2007). IHHNV infection in $L$. vannamei does not lead to death, but causes 'runt deformity syndrome' (Chayaburakul et al., 2005). Therefore, IHHNV is a suitable virus to study the immune response in $L$. vannamei against viral infection, due to the convenience for long time observation for shrimp regardless of their mortality. WSSV is the most serious pathogen for shrimp. In cultured shrimp, WSSV can cause an accumulative mortality of 100\% within 3-10 days (Lightner et al., 1997). In this study, the mRNA expression levels of LvRab5B, LvRab6A, and LvRab7 were tested in the hepatopancreas of L. vannamei at different stages after IHHNV and WSSV challenge using qRT-PCR. The results showed that LvRab5B, LvRab6A, and LvRab7 were significantly up-regulated during 48-84 h post injection. These results suggest that LvRab5B, LvRab6A, and LvRab7 may participate in the shrimp immune response to viral infection. Our finding is consistent with previous studies showing that Rab7 is involved in the anti-viral response in Penaeus monodon. Previous studies revealed that the P. monodon Rab7 (PmRab7) protein can combine with a viral envelope, VP28, of the WSSV to avoid lysosomal digestion (Sritunyalucksana et al., 2006). Several studies also demonstrated that silencing of PmRab7 by injecting double-stranded RNA corresponding to PmRab7 (dsRNA-PmRab7) can effectively prevent WSSV, TSV, or yellow head virus infection, suggesting that Rab7 is involved in the endosomal trafficking pathway of virus infected penaeid shrimp (Ongvarrasopone et al., 2008, 2011; Thagun et al., 2012). The results in the present study provide further evidence that LvRab7 is involved in the antiviral activity of penaeid shrimp against IHHNV and WSSV infection. However, whether the Rab7 protein shares a similar antiviral mechanism with different viruses remains to be investigated in more depth.

In conclusion, we cloned the full-length cDNA sequences of LvRab5B, LvRab6A, and LvRab7 from $L$. vannamei for the first time, and revealed that these genes may play critical roles in the immune response to viral infection in shrimp, which will help to further our understanding of the immune system of shrimp.

\section{ACKNOWLEDGMENTS}

Research supported by the National High Technology Research and Development Program of China (\#2012aa10a404), the Natural Science Foundation of Guangxi (\#2012gxnsfda053015), the Modern Agricultural Technology System Specific (cars-47), and the National Modern Agriculture Industry Technology System Project (\#nycytxgxcxtd-03-14).

\section{Conflicts of interest}

The authors declare no conflict of interest. 


\section{REFERENCES}

Bourne HR, Sanders DA and McCormick F (1990). The GTPase superfamily: a conserved switch for diverse cell functions. Nature 348: 125-132.

Chayaburakul K, Lightner DV, Sriurairattana S, Nelson KT, et al. (2005). Different responses to infectious hypodermal and hematopoietic necrosis virus (IHHNV) in Penaeus monodon and P. vannamei. Dis. Aquat. Organ. 67: 191-200.

Diekmann Y, Seixas E, Gouw M, Tavares-Cadete F, et al. (2011). Thousands of rab GTPases for the cell biologist. PLoS Comput. Biol. 7: e1002217.

Hutagalung AH and Novick PJ (2011). Role of Rab GTPases in membrane traffic and cell physiology. Physiol Rev. 91: 119-149.

Jordens I, Westbroek W, Marsman M, Rocha N, et al. (2006). Rab7 and Rab27a control two motor protein activities involved in melanosomal transport. Pigment Cell Res. 19: 412-423.

Lightner DV, Redman RM, Poulos BT, Nunan LM, et al. (1997). Risk of spread of penaeid shrimp viruses in the Americas by the international movement of live and frozen shrimp. Rev. Sci. Tech. 16: 146-160.

Martin GG, Simcox R, Nguyen A and Chilingaryan A (2006). Peritrophic membrane of the penaeid shrimp Sicyonia ingentis: structure, formation, and permeability. Biol. Bull. 211: 275-285.

Ongvarrasopone C, Chanasakulniyom M, Sritunyalucksana K and Panyim S (2008). Suppression of PmRab7 by dsRNA inhibits WSSV or YHV infection in shrimp. Mar.Biotechnol. 10: 374-381.

Ongvarrasopone C, Saejia P, Chanasakulniyom M and Panyim S (2011). Inhibition of Taura syndrome virus replication in Litopenaeus vannamei through silencing the LvRab7 gene using double-stranded RNA. Arch. Virol. 156: 1117-1123.

Pereira-Leal JB and Seabra MC (2001). Evolution of the Rab family of small GTP-binding proteins. J. Mol. Biol. 313: 889-901.

Prior S, Browdy CL, Shepard EF, Laramore R, et al. (2003). Controlled bioassay systems for determination of lethal infective doses of tissue homogenates containing Taura syndrome or white spot syndrome virus. Dis. Aquat. Organ. 54: 89-96.

Robalino J, Almeida JS, McKillen D, Colglazier J, et al. (2007). Insights into the immune transcriptome of the shrimp Litopenaeus vannamei: tissue-specific expression profiles and transcriptomic responses to immune challenge. Physiol. Genomics 29: 44-56.

Saito-Nakano Y, Loftus BJ, Hall N and Nozaki T (2005). The diversity of Rab GTPases in Entamoeba histolytica. Exp. Parasitol. 110: 244-252.

Segev N (2001). Ypt/rab gtpases: regulators of protein trafficking. SCi STKE 2001: re11.

Sritunyalucksana K, Wannapapho W, Lo CF and Flegel TW (2006). PmRab7 is a VP28-binding protein involved in white spot syndrome virus infection in shrimp. J. Virol. 80: 10734-10742.

Tang KF, Navarro SA and Lightner DV (2007). PCR assay for discriminating between infectious hypodermal and hematopoietic necrosis virus (IHHNV) and virus-related sequences in the genome of Penaeus monodon. Dis. Aquat. Organ. 74: 165-170.

Thagun C, Srisala J, Sritunyalucksana K, Narangajavana J, et al. (2012). Arabidopsis-derived shrimp viral-binding protein, PmRab7 can protect white spot syndrome virus infection in shrimp. J. Biotechnol. 161: 60-67.

Valles-Jimenez R, Cruz P and Perez-Enriquez R (2004). Population genetic structure of Pacific white shrimp (Litopenaeus vannamei) from Mexico to Panama: microsatellite DNA variation. Mar. Biotechnol. 6: 475-484.

Zeng D, Chen X, Xie D, Zhao Y, et al. (2013). Transcriptome analysis of Pacific white shrimp (Litopenaeus vannamei) hepatopancreas in response to Taura syndrome virus (TSV) experimental infection. PLoS One 8: e57515.

Zhang M, Chen L, Wang S and Wang T (2009). Rab7: roles in membrane trafficking and disease. Biosci. Rep. 29: 193-209. 